Haematology, The Royal Children's Hospital, Melbourne, Vic, Australia; ${ }^{4}$ Cancer and Haematology Division, Walter and Eliza Hall Institute, Parkville, Vic, Australia; ${ }^{5}$ Department of Medicine, The University of Melbourne, Parkville, Vic, Australia

Contact Dr Emma Leitinger.

E-mail: Leitingerej@gmail.com

1. Chan K, Blombery $\mathrm{P}$, Jones $\mathrm{K}$, et al. Plasmablastic Richter transformation as a resistance mechanism for chronic lymphocytic leukaemia treated with BCR signalling inhibitors. Br J Haematol 2017; 177: 319-30.

2. Feldman AL, Arber DA, Pittaluga S, et al. Clonally related follicular lymphomas and histiocytic/dendritic cell sarcomas: evidence for transdifferentiation of the follicular lymphoma clone. Blood 2008; 111: 5433-9.

3. Hure MC, Elco CP, Ward D, et al. Histiocytic sarcoma arising from clonally related mantle cell lymphoma. J Clin Oncol 2012; 30: e49-53.

4. Shao H, Xi L, Raffeld M, et al. Clonally related histiocytic/dendritic cell sarcoma and chronic lymphocytic leukemia/small lymphocytic lymphoma: a study of seven cases. Mod Pathol 2011; 24: 1421-32.

5. Juskevicius D, Dirnhofer S, Tzankov A. Genetic background and evolution of relapses in aggressive B-cell lymphomas. Haematologica 2017; 102: 1139-49.

6. Agarwal RCY, Chan Y-C, Tam CS, et al. Dynamic molecular monitoring reveals that SWI-SNF mutations mediate resistance to ibrutinib plus venetoclax in mantle cell lymphoma. Nat Med 2019; 25: 119-29.
7. Herling CD, Abedpour N, Weiss J, et al. Clonal dynamics towards the development of venetoclax resistance in chronic lymphocytic leukemia. Nat Commun 2018; 9: 727.

DOI: https://doi.org/10.1016/j.pathol.2021.01.013

\section{Lymphocyte nuclei of nodal marginal zone lymphoma mimicking granulocytic morphology with Pelger-Huët-like features}

Sir,

Nodal marginal zone lymphoma (NMZL) with peripheral blood involvement is observed in only a small proportion of patients and usually shows small-to medium-sized centrocyte-like neoplastic lymphocytes in a peripheral blood smear. $^{1-3}$ This disease may present with frequent plasmacytoid differentiation or occasional monocytoid appearance; however, the granulocytoid appearance of nuclei is rarely
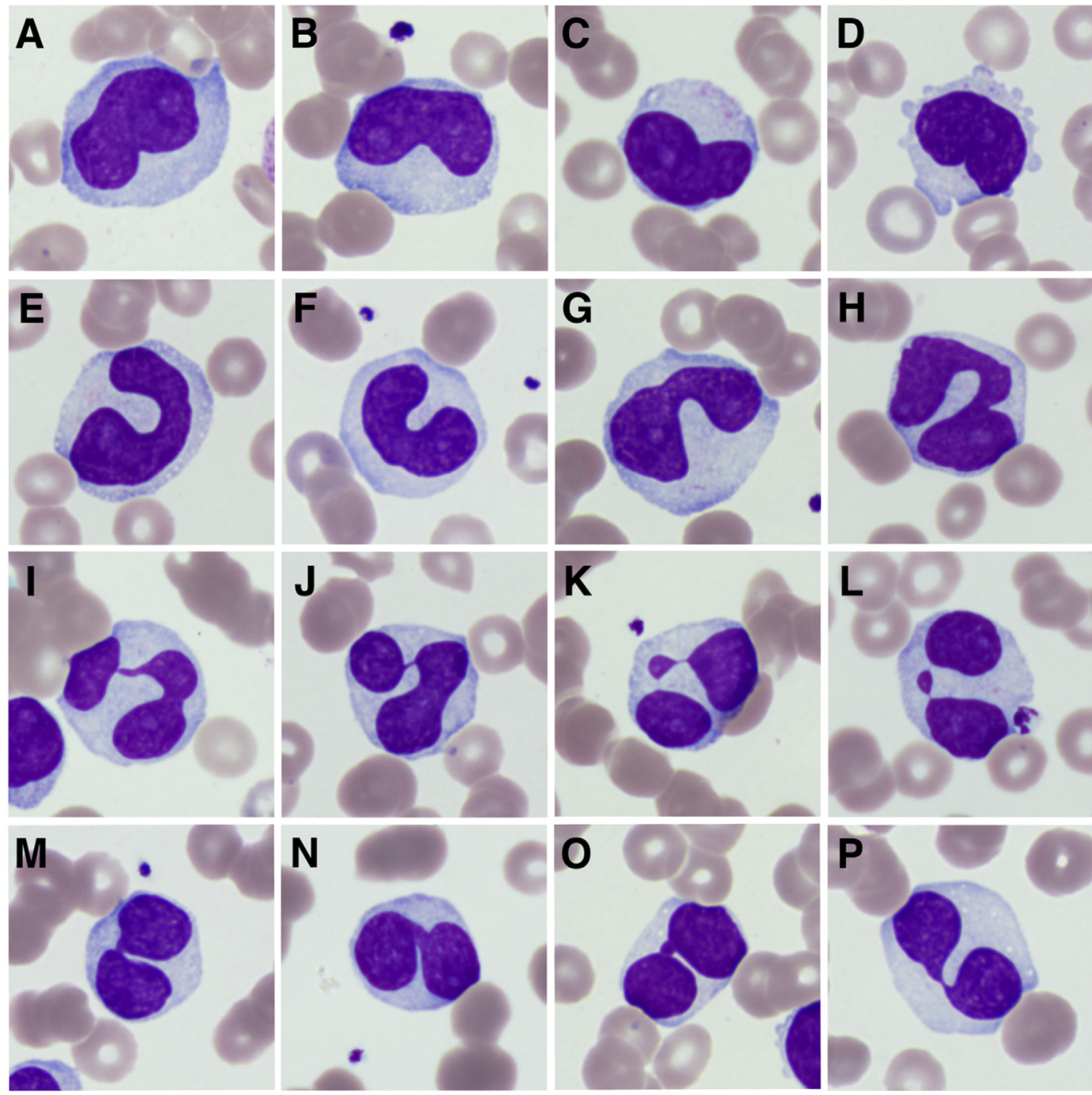

Fig. 1 Morphology of abnormal lymphocytes on peripheral smear. (A-D) Metamyelocyte-like kidney bean-shaped nuclei; (E-H) band-shaped nuclei; (I-L) multilobed nuclei; and $(\mathrm{M}-\mathrm{P})$ pseudo Pelger-Huët-like pince-nez-shaped nuclei (Wright-Giemsa staining $\times 1000$ ). 
observed. Here, we report a case of NMZL with a granulocytoid appearance of nuclei and a Pelger-Huët-like anomaly in a peripheral blood smear.

A 56-year-old female presented with neck lymphadenopathy and night sweats for 4 months. Her complete blood count demonstrated haemoglobin $88 \mathrm{~g} / \mathrm{L}$, lymphocytes $22.73 \times 10^{9}$ / $\mathrm{L}$ and platelets $156 \times 10^{9} / \mathrm{L}$. The blood film revealed $78 \%$ abnormal lymphocytes, which mimicked granulocytic development in nuclear morphology, including metamyelocyte-like kidney bean-shaped nuclei (Fig. 1A-D), band-shaped nuclei (Fig. 1E-H) and multilobed nuclei (Fig. 1I-L). It was interesting to note that some neoplastic lymphocytes showed pseudo Pelger-Huët-like pince-nez-shaped nuclei (Fig. 1M$\mathrm{P})$. Flow cytometric analysis performed on bone marrow revealed the presence of an atypical lymphocyte population that comprised $83 \%$ of the total lymphocytes analysed. The atypical lymphocytes expressed CD19, CD22, lambda, and CD200 and partially expressed CD23 but lacked CD5, CD10, CD103, kappa, CD138, CD38, CD34, and sIgM. Molecular study revealed $B I R C 3$ and $P 53$ mutations. Bone marrow and lymph node biopsies confirmed the diagnosis of NMZL with bone marrow infiltration.

Pelger-Huët anomaly is an inherited benign condition that is characterised by the abnormality of chromosome segregation of neutrophils leading to bilobed, peanut- or dumbbellshaped nuclei. The classic morphological abnormality is known to be caused by mutations of the Lamin B receptor (LBR) gene, which maintains the nuclear membrane structure. ${ }^{4}$ Pseudo Pelger-Huët anomaly, as a marker of granulocytic dysplasia, is only involved in neutrophils, which may occur in myelodysplastic syndrome (MDS) and acute and chronic leukaemias or can be drug-induced. ${ }^{5,6}$ The PelgerHuët-like anomaly of lymphocytes is an extremely uncommon phenomenon that, to the best of our knowledge, has not been reported before. In contrast to the neutrophil PelgerHuët anomaly, which is caused by failure of nuclear segmentation, the lymphocyte Pelger-Huët-like anomaly is caused by abnormal nuclear lobulation.

Conflicts of interest and sources of funding: This work was funded by National Natural Science Foundation of China (No. 81702070), China; CAMS Innovation Fund for Medical Sciences (CIFMS) (No. 2018-12M-AI-002) and Beijing Key Clinical Specialty for Laboratory Medicine - Excellent Project (No. ZK201000), China. The authors state that there are no conflicts of interest to disclose.

\section{Fei Wang*, Geng Wang*, Wei Wu}

Department of Laboratory Medicine, Peking Union Medical College Hospital, Peking Union Medical College and Chinese Academy of Medical Sciences, Beijing, China; * these authors contributed equally to this work

Contact Wei Wu.

E-mail:ww_pumch@sina.com

1. Camacho FI, Algara P, Mollejo M, et al. Nodal marginal zone lymphoma: a heterogeneous tumor: a comprehensive analysis of a series of 27 cases. Am J Surg Pathol 2003; 27: 762-71.
2. Inamdar KV, Medeiros LJ, Jorgensen JL, Amin HM, Schlette EJ. Bone marrow involvement by marginal zone B-cell lymphomas of different types. Am J Clin Pathol 2008; 129: 714-22.

3. Traverse-Glehen A, Felman P, Callet-Bauchu E, et al. A clinicopathological study of nodal marginal zone B-cell lymphoma. A report on 21 cases. Histopathology 2006; 48: 162-73.

4. Nikolakaki E, Mylonis I, Giannakouros T. Lamin B receptor: interplay between structure, function and localization. Cells 2017; 6: 28.

5. Roberts T, Linenberger M. A case of benign Pelger-Huet anomaly. Blood 2015; 126: 693

6. Cunningham JM, Patnaik MM, Hammerschmidt DE, Vercellotti GM Historical perspective and clinical implications of the Pelger-Huet cell. Am J Hematol 2009; 84: 116-9.

DOI: https://doi.org/10.1016/j.pathol.2021.02.014

\section{Micronodular thymoma with lymphoid stroma: a clinicopathological study of five cases}

Sir,

Micronodular thymoma with lymphoid stroma (MTLS) is a rare subtype of thymoma that was a new inclusion in the third edition of the World Health Organization (WHO) Classification of Tumours of the Lungs, Pleura, Thymus and Heart released in 2004. ${ }^{1}$ Recognition of this histological subtype of thymoma is important as it has an excellent prognosis, with only a single published case of recurrence that occurred after incomplete initial resection. ${ }^{2}$

We identified all MTLS from the archives of Tissue $\mathrm{Pa}$ thology and Diagnostic Oncology, Royal Prince Alfred Hospital, NSW Health Pathology, Sydney, and reviewed clinical and pathological features. Over an 8-year time period, five cases of MTLS were identified.

The patients were four women and one man, aged between 58 and 70 years (mean 65.4 years) (Table 1). All tumours were found incidentally on computed tomography within the thymus in the anterior mediastinum and there was no evidence of regional nor metastatic spread. None of the patients had symptoms consistent with myasthenia gravis or any other autoimmune diseases. All patients underwent thymectomy, one with a minimally invasive surgical technique with the rest via conventional midline sternotomy. Two patients had cardiac valve pathology requiring intervention, which was attended to at the same time as thymectomy.

Tumour size ranged between 22 and $60 \mathrm{~mm}$ in maximum dimension (mean $45.4 \mathrm{~mm}$ ). One tumour was solid, with the rest mixed solid and cystic. There were similar histological findings in all tumours (Fig. 1A-D). The tumours were well circumscribed and one tumour had a variable thickness capsule, with the rest unencapsulated. There were coalescing nests of bland oval to elongated epithelial cells with an intervening rich lymphoid stroma. The epithelial cells showed oval vesicular nuclei, small nucleoli and moderate amounts of indistinct pale eosinophilic cytoplasm. The lymphoid stroma contained follicles with reactive germinal centres. Up to four lymph nodes were identified within the 